\title{
Chaotic Dynamics and Simulation of Japanese Vowel Sounds
}

\author{
Michael Small * $\quad$ Chi Kong Tse* Tohru Ikeguchi ${ }^{\dagger}$
}

\begin{abstract}
We examine deterministic dynamics in Japanese speech vocalisation patterns. We find that a simple data driven non-parametric model may be employed to accurately and compactly describe the complex dynamic structure of the data. Moreover, we demonstrate that the data is not consistent with a monotonic nonlinear transformation of linearly filtered noise (i.e. determinism in the data cannot be adequately described by the Fourier power spectrum). We then show that for four of the five vowel sounds, the dynamics are not consistent with a noise driven nonlinear periodic orbit, and therefore the underlying dynamical system is likely to be chaotic. In the fifth case (the vowel $/ u /$ ) we are unable to reject the hypothesis of a noise driven periodic orbit.
\end{abstract}

\section{INTRODUCTION}

Time series recordings of human vocalisation patterns exhibit complex dynamics over a variety of different time scales. It is tempting to characterise such data using the Fourier power spectrum. In this paper we show that this is not sufficient and suggest a viable alternative. We demonstrate potential application of this technique for simulation of individual vowel sounds and also suggest a potential method for speaker verification. Moreover, in four out of five cases we conclude that the deterministic dynamics underlying these vocalisation patterns are aperiodic, bounded and deterministic: sufficient conditions for the existence of chaos. In the fifth case $(/ \mathrm{u} /)$ we fail to find sufficient evidence to reject the hypothesis that the data is a noise-driven periodic orbit

Human vocalisation patterns are, virtually by definition, approximately periodic $[1,2]$. In [2] Ikeguchi and colleagues presented an analysis of vowel sounds using the methods of nonlinear dynamical system theory (at that time, this meant correlation dimension [3] and linear surrogate tests [4]). In this paper we repeat this analysis: using new statistics and alternative tests. We conclude that the data is not consistent with linearly filtered noise (the same result at [2]). This result, however, does not distinguish between the two most

\footnotetext{
*Department of Electronic and Information Engineering, Hong Kong Polytechnic University. e-mail: [ensmall, encktse] @polyu . edu.hk

$\dagger$ Graduate School of Science and Engineering, Saitama University, Saitama, Japan. e-mail tohru@nls.ics.saitama-u.ac.jp.
}

likely alternative hypotheses: a noise-driven periodic orbit, or bounded aperiodic dynamics. A new surrogate test [5], capable of distinguishing between noise-driven periodic orbits and pseudoperiodic chaos has been suggested. This algorithm has been applied to reconstruct attractors for various vowel sounds [6]. In this paper we apply this method to distinguish noise-driven periodic orbits from chaos. Our conclusions show that four of five vowel sounds we consider are chaotic.

Although we choose to analyse Japanese vowel sounds, there is essentially nothing to motivate this decision. The same techniques could equally be applied to speech sounds in other languages.

Simulation of voice patterns can be achieved relatively easily by using pre-recorded samples, possibly by modulating the signal to mimic a particular speaker. Alternatively, neural networks, radialbasis functions or one of many possible curve fitting techniques can be applied to estimate the underlying dynamics and then used to simulate speech samples. These techniques are computationally very expensive and highly parametric. We provide an alternative surrogate based technique and show that such simulations are easy to obtain and closely match the data. This technique may also be adapted as a form of speaker identification, using the vowel sound pronunciation as a form of "vocal fingerprint": surrogate data methods provide an estimate of confidence in the identification.

\section{DATA}

We consider recordings of five Japanese vowel sounds (/a/, /i/, /u/, /e/ and /o/), as vocalised by a male subject and sampled at $48 \mathrm{kHz}$. We analyse a short segment of 25000 data (approximately 0.5 sec.) obtained from the mid-point of the recording. Typical data, over a variety of time scales is shown in Fig. 1. The Japanese vowel sounds; /a/, /i/, $/ \mathrm{u} / \mathrm{e} /$ and $/ \mathrm{o} /$; are very similar in appearance to the same five English vowels.

\section{ALGORITHMS}

We employ four basic tools from the nonlinear time series analysis toolkit: the Gaussian kernel algorithm (GKA) for correlation dimension estima- 

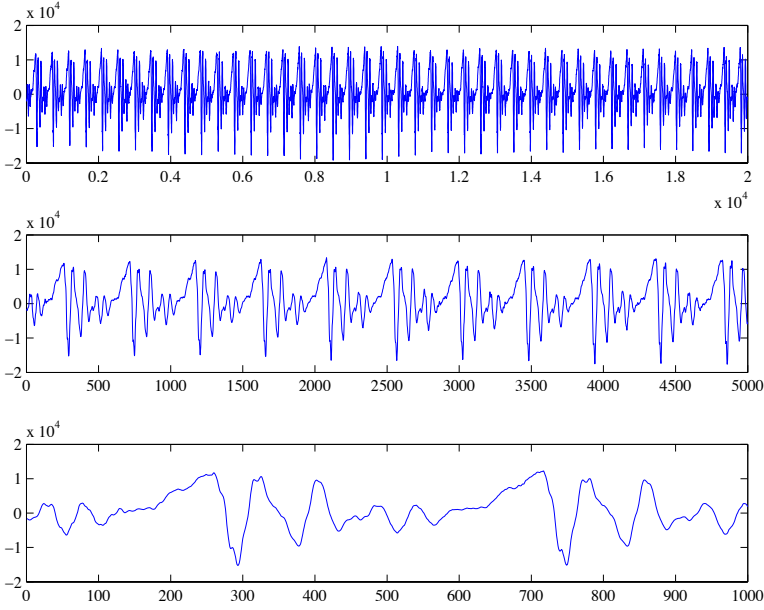

Figure 1: Vowel sound data for the vowel /a/, sampled at $48 \mathrm{kHz}$. Shown are samples of length 20000 , 5000 and 1000.

tion (Sec. 3.1); an estimator of algorithmic complexity (Sec. 3.2); linear surrogate analysis (Sec. 3.3 ); and, the pseudo-periodic surrogate (PPS) algorithm (Sec. 3.4). The GKA and algorithmic complexity are statistical measures of the structure and complexity evident in a time series. In the current study we use these algorithms as quantitative measures to compare data and simulated time series. The surrogate algorithms are techniques that generate simulated time series both very "like" the original data, and also consistent with some specific hypothesis. By comparing data and surrogates, with some quantitative measure, we are able to determine whether the data is also typical of that hypothesis. In the remainder of this section we describe each of these four techniques.

\subsection{The Gaussian Kernel Algorithm}

Although the original algorithm, suggested by Grassberger and Proccacia in 1983 [3], has since been found to have some problems, estimation of correlation dimension is still common place. Two common objections to the Grassberger-Proccacia algorithm (GPA) is that it does not properly account for finite data or observational noise. We use an alternative algorithm, the Gaussian Kernel Algorithm (GKA) which attempts to address both of these problems by accounting for the effect of additive Gaussian observational noise [7]. Essentially, the standard GPA estimates the correlation dimension by considering the distribution of interpoint distances (or correlation integral)

$$
C_{N}(\epsilon)=P\left(\left\|z_{i}-z_{j}\right\|<\epsilon\right)
$$
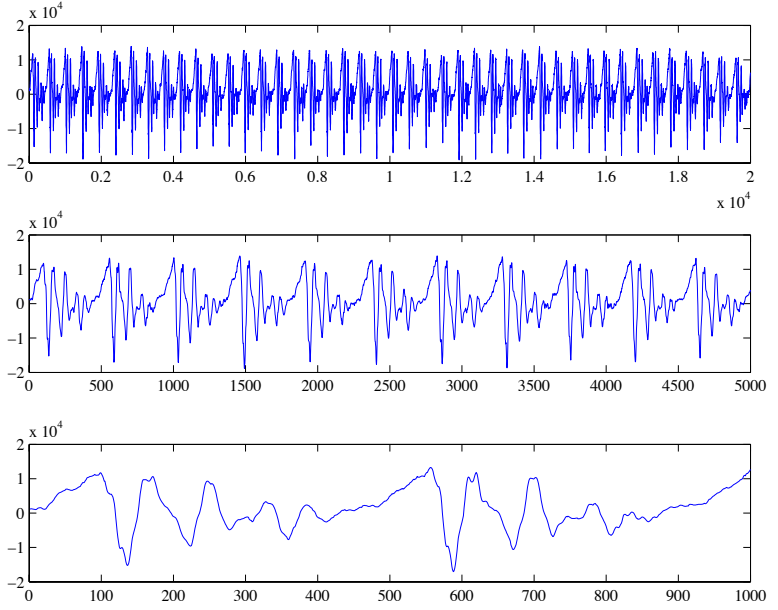

Figure 2: Nonlinear PPS data generated for the vowel data in Fig. 1. The three plots are of the same lengths as the data shown in Fig. 1.

where $z_{i}$ and $z_{j}$ are vector points obtained by a time delay embedding of the scalar time series $\left\{x_{i}\right\}$ according to $z_{i}=\left(x_{i}, x_{i-\tau}, x_{i-2 \tau}, \ldots, x_{i-\left(d_{e}-1\right) \tau}\right)$. The GPA estimates the probability $P\left(\left\|z_{i}-z_{j}\right\|<\epsilon\right)$ by simply computing the histogram directly from the data. In contrast the GKA assumes that noise in $z_{i}$ introduces uncertainty in $P\left(\left\|z_{i}-z_{j}\right\|<\epsilon\right)$ and instead computes

$$
T_{N}^{\left(d_{e}\right)}(h)=\frac{1}{N(N-1)} \sum_{\substack{0 \leq i, j \leq N \\ j \neq i}} e^{\frac{-\left\|z_{i}-z_{j}\right\|^{2}}{4 h^{2}}}
$$

where the bandwidth $h$ takes the role of $\epsilon$. It follows that $T_{N}^{\left(d_{e}\right)}(h)$ scales according to [8]

$$
T_{N}^{\left(d_{e}\right)}(h) \propto\left(\frac{e^{-K \tau} h^{2}}{h^{2}+\sigma^{2}}\right)^{d_{e}}\left(\frac{h^{2}+\sigma^{2}}{d_{e}}\right)^{\frac{d_{c}}{2}} .
$$

A nice benefit of this algorithm is that, in addition to the correlation dimension $d_{c}$, one is also able to estimate both $K$, the correlation entropy; and $\sigma^{2}$, the noise variance. The results we present in this paper are estimates of $K$ rather than correlation dimension or noise. The reason for this is that correlation dimension itself is not a sensitive enough measure to differentiate between data and surrogates.

\subsection{Algorithmic Complexity}

We employ algorithmic complexity as a second independent test statistic. Algorithmic complexity is essentially an estimate of the compression one could achieve for a given symbolic sequence using a Lempel-Ziv syntactic compression scheme (see [9]). 
To compute this quantity from a time series, one first has to extract a symbolic sequence. We do this by transforming each scalar data $x_{i}$ to correspond to one of four different symbols $b^{(1)}, b^{(2)}$, $b^{(3)}$, or $b^{(4)}$. We partition the data so that the probability of each of these symbols occurring is equal, and then apply the transformation $x_{i} \rightarrow b_{i}$ where $b_{i} \in\left\{b^{(1)}, b^{(2)}, b^{(3)}, b^{(4)}\right\}$. Finally, we apply the Lempel-Ziv algorithm to compute the number of unique symbol sequences. This quantity is then normalised by the expected number of unique symbols for a random sequence [9]. A complexity near 0 means that the time series is completely deterministic and periodic (a stable fixed point, with no noise). A complexity approaching 1 means that the signal is highly random. Chaotic sequences occupy the middle ground.

\subsection{Linear surrogate tests}

Surrogate tests provide a method to compare observed time series data with data which are consistent with some specified hypothesis and also like the observed data. A quantitative comparison (using one of the statistics described previously) yields two possibilities: if the statistic values for the data are typical of the surrogates, then one is unable to reject the hypothesis (i.e. the hypothesis may be true); alternatively, if the statistic values for the data are atypical we can reject the hypothesis as false. Of the three commonly applied surrogate algorithms [4], we employ the amplitude adjusted Fourier transform algorithm (aka the AAFT). This algorithm generates artificial data which test the hypothesis of a monotonic nonlinear transformation of linearly filtered noise as an origin of the data. Rejection of this hypothesis indicates that the Fourier power spectrum does not offer a complete description of the deterministic dynamics in the data.

\subsection{Pseudo-periodic surrogate data}

The AAFT surrogate algorithm tests data against the hypothesis of linear noise (albeit transformed by a static nonlinear filter). For the vowel data exhibited in Fig. 1, this may not be sufficient. We therefore employ the pseudo-periodic surrogate algorithm (PPS) as well.

The PPS algorithm is employed here for two distinct purposes: (i) to test whether the data may be described by a stable periodic orbit with dynamic noise (i.e. a noise driven periodic orbit), and (ii) to generate artificial data which are indistinguishable from the true data. The algorithm is described fully elsewhere [9], but may be summarised as fol-

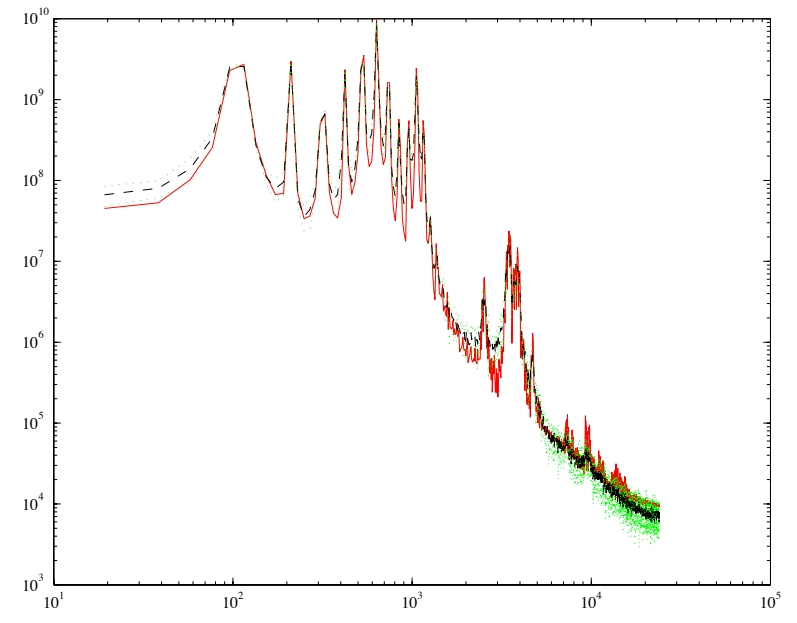

Figure 3: Frequency domain comparison of the data and simulation shown in Figs. 1 (solid) and 2 (dashed).

lows. Scalar data are embedded to produce a vector time series $\left\{z_{i}\right\}$ representative of the underlying dynamics. The surrogate data is then generated by randomly selecting a point in $\left\{z_{i}\right\}_{i=1}^{N}$. A sequence of embedding points is then generated by choosing a neighbour $z_{j}$ of the currently selected point $z_{i}\left(\left\|z_{i}-z_{j}\right\|\right.$ is small) and determining its successor $z_{j+1}$. That successor becomes the new current point and the procedure repeats.

The parameter $\rho$ determines how to choose the neighbours at each step [5]. We define a related parameter $\hat{\rho}=P\left(x_{i}=x_{j}\right)$. Then, for small values of $\hat{\rho}$ it is very likely that $z_{j}=z_{i}$ and therefore the data very closely follow the original. For large $\hat{\rho}$ it is more likely that $\left\|z_{i}-z_{j}\right\|$ is large and the two points are unrelated. By tuning $\hat{\rho}$ we may select the neighbours so that all the underlying dynamics is preserved: in this case $\hat{\rho}$ and the random selection of neighbours merely mimics a new realisation of the dynamic noise in the system. Alternatively, we may select neighbours so that the general periodic structure is all that is preserved: in this case the artificial data are surrogates addressing the hypothesis of a noise driven periodic orbit.

We use the second scenario to test whether this vowel vocalisation data may be described as a noisy periodic orbit. We use the first scenario to generate artificial data indistinguishable from the original. Figure 2 illustrates the generation of artificial data which is distinct from, but qualitatively indistinguishable from the original data (Fig. 1). In Fig. 3 we provide a frequency domain comparison for these two data sets. 

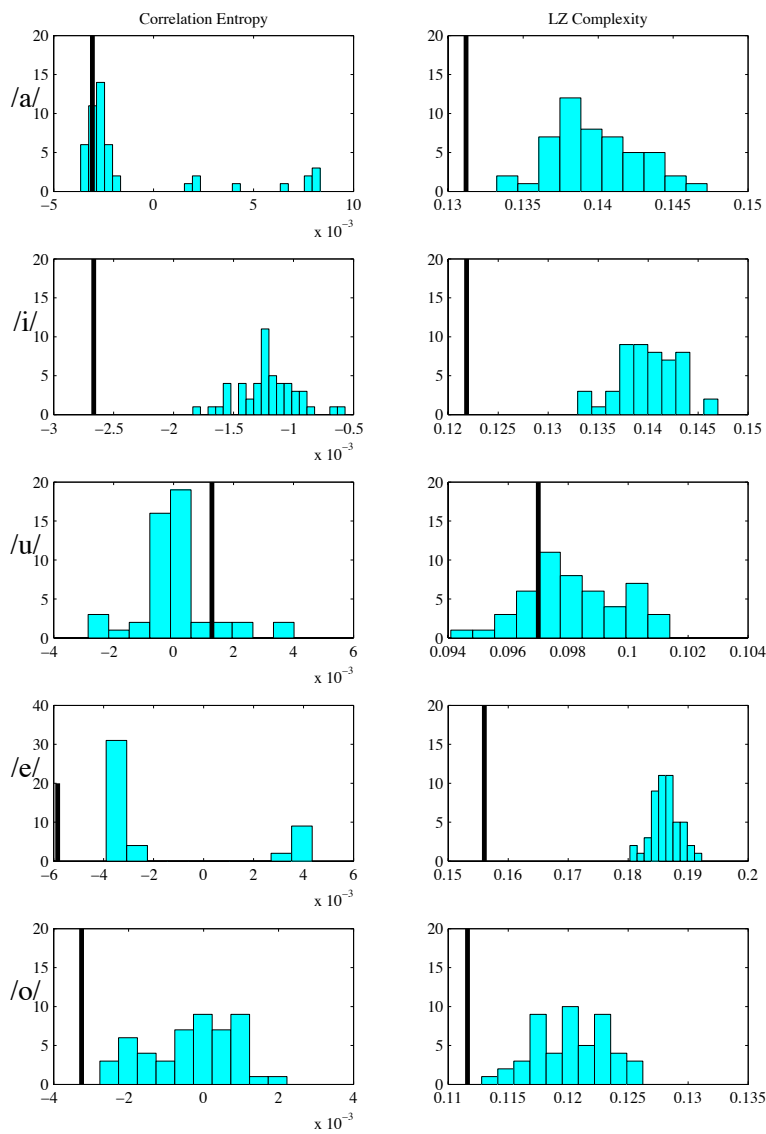

Figure 4: Surrogate data calculations for each of the five vowel sound data sets. In each case, the left panel is a comparison of the correlation entropy (computed from the GKA) for $\left(d_{e}=8\right.$ and $\tau$ selected at the firs zero of the autocorrelation), the right panel is the algorithmic complexity. The histogram is computed values for 50 periodic surrogates, the solid line is for the data. In all cases except $/ \mathrm{u} /$, the data and surrogates are clearly distinct.

\section{RESULTS AND CONCLUSIONS}

We first consider the AAFT surrogate algorithm, and use it to discount the possibility that this data can be described as a linear noise process. For each of the five data sets, we compute correlation dimension and entropy using the GKA and complexity using the algorithm briefly described in Sec. 3.2. We compare these statistics to the same quantities estimated from 50 AAFT surrogate data sets. In each case, the entropy and complexity of the true data is less than all 50 surrogates. We can therefore reject at the $98 \%$ confidence level the possibility that these data are consistent with linear noise.

The main purpose of this paper is to apply the PPS algorithm and generate data indistinguishable from the original data. In Fig. 2 we demonstrate that this can be easily achieved. For each data set we embed the data with $d_{e}=8$ and $\tau$ selected at the first zero of the autocorrelation function [9]. The PPS algorithm, with $\hat{\rho}=0.1$, then yields surrogates which exhibit qualitative features and Fourier power spectra indistinguishable from the true data. Fig. 3 illustrates the power spectra for the data in Figs. 1 and 2.

This is sufficient to illustrate the application of the PPS scheme for accurate simulation of the data. We now also use it to determine the dynamic origin of this data. For each of the six data sets we compute 50 surrogates using the PPS scheme and compare estimates of correlation dimension, correlation entropy and algorithmic complexity. In Fig. 4 we see that for each of the vowel sounds /a/ /i/, /e/ and /o/ the dynamics are distinct from a noise driven periodic orbit. For the data from $/ \mathrm{u} /$ we cannot draw this conclusion.

\section{Acknowledgments}

The work described in this paper was fully supported by a grant from the Research Grants Council of Hong Kong (Project No. PolyU 5235/03E).

\section{References}

[1] R. Tokunaga et al. A simple geometrical structure underlying speech signals of the japanese vowel /a/. Intl J Bif Chaos, 6:149-160, 1996.

[2] T. Ikeguchi and K. Aihara. Estimating correlation dimensions of biological time series with a reliable method. J Intelligent Fuzzy Systems, 5:33-52, 1997.

[3] P. Grassberger and I. Procaccia. Characterization of strange attractors. Physl Rev Lett, 50:346-349, 1983.

[4] J. Theiler et al. Testing for nonlinearity in time series: The method of surrogate data. Physica D, 58:77-94, 1992.

[5] M. Small et al. A surrogate test for pseudo-periodic time series data. Phys Rev Lett, 87:188101, 2001.

[6] Z. Li et al. Nonlinear analysis of Chinese vowels. In Proceedings of the 2002 IEICE General Conference, page 57, Waseda University, Japan, 2002. In Japanese.

[7] D.J. Yu et al. Efficient implementation of the Gaussian kernel algorithm in estimating invariants and noise level from noisy time series data. Phys Rev E, 61:3750-3756, 2000.

[8] C. Diks. Estimating invariants of noisy attractors. Phys Rev E, 53:R4263-R4266, 1996.

[9] M. Small. Applied Nonlinear Time Series Analysis: Applications in Physics, Physiology and Finance, volume 52 of Nonlinear Science Series A. World Scientific, Singapore, 2005. 